\title{
Viable Adrenal Medullary Transplants in Non-Human Primates: Increasing the Number of Grafts
}

\author{
Mark Dubach \\ Department of Psychiatry and Behavioral Sciences \\ and \\ Regional Primate Research Center \\ University of Washington \\ Seattle, Washington, USA
}

\section{SUMMARY}

The robust survival of stereotaxic adrenal medullary autografts in monkey brain parenchyma depends heavily on technique $/ 11 /$. One aspect of technique critical for clinical applications of CNS grafting is the problem of spreading treatment effects throughout large regions in a primate brain. Stereotaxic placement of a large number of grafts, which would address this problem, would require that later grafts retain the capacity for viability over a long period of time during the surgery, while earlier grafts are being made. In the present experiments involving 12 longtailed macaques (Macaca fascicularis), some grafts remained in medium $2.5 \mathrm{~h}$ before being transplanted, without apparent loss of viability.

A clinically sufficient distribution of graft tissue would also require a large number of separate grafts to be derived from one adrenal medulla. One method for subdividing the gland might be to cut it into numerous very small pieces, but various dicing techniques described in this report have yielded little viable graft tissue. On the other hand, the number of ribbon grafts of the original dimensions available from a single gland is rather small; adrenal ribbons of the original dimensions were therefore further dissected. Half-length and half-width ribbons were successful, but less so than full-size ribbons in terms of

Reprint address:

Mark Dubach

Regional Primate Reseach Center

University of Washington SJ-50

Seattle, WA 98195

USA proportionate viability. An assay of behavioral effectiveness, applied to all subjects described here, is presented in a separate contribution /9/.

\section{KEY WORDS}

transplantation, adrenal medulla, monkey, parkinsonism, neostriatum, substantia nigra, CNS grafting

\section{INTRODUCTION}

Transplantation as a treatment for Parkinson's disease and its animal models was conceived as a means of providing catecholamine for denervated dopamine terminal areas $/ 5,19,31 /$. Few clinical surgeries, however, have attempted to distribute transplant tissue throughout the neostriatum /15/; most have placed diced autologous adrenal medullary tissue in a cavity in the ventricular aspect of the caudate nucleus $/ 26,27 /$. Viability of adrenal grafts, moreover, has been minimal in human patients at autopsy $/ 3,14,23,24,29 /$, and in early non-human primate recipients $/ 4,20,28 /$. The original concept of transplantation as a neurotransmitter replacement therapy has not been tested by these surgeries. Rodent experiments have often indicated that grafts are more viable at ventricular or cavitation sites than at intraparenchymal sites $/ 17 /$, and that fetal nigral tissue is more effective than adrenal autograft tissue $/ 18 /$; because of the small size of the rodent brain, these results are consistent with a neurotransmitter replacement interpretation, particularly for 
nigral grafts, which can reinnervate a significant portion of the striatum /16/. A test of catecholamine replacement as a therapy in primates, however, will require new methods adapted to larger brains.

We recently developed techniques for making viable intraparenchymal adrenal grafts in nonhuman primate brains $/ 8,11 /$. The grafts retained tyrosine hydroxylase immunoreactivity and glandular morphology for at least eight weeks. Since these methods differed substantially from those previously employed in clinical or animal experiments, it may be that viability depends as much on transplantation technique as on graft site, host species, or source of tissue. Intraparenchymal adrenal medullary grafts in monkeys may represent a feasible method for distributing grafts and testing the catecholamine replacement concept in a large-brained animal.

The present experiments test two aspects of increasing the number of grafts: (i) increasing the number of grafts available from an adrenal gland by dividing larger ribbons into smaller ribbons, and (ii) leaving a long interval between adrenal ligation and ribbon transplantation so that multiple grafts can be made in sequence. Histological evidence indicates that downsizing adrenal ribbons by further dissection decreases proportional viability, but that a prolonged incubation in vehicle at room temperature may even increase viability. Histological results of these experiments and those already reported /11/ are compared with results of earlier transplants employing a variety of tissue-fragment techniques.

\section{MATERIALS AND METHODS}

\section{Subjects}

Graft techniques were developed in a series of 24 surgeries on young adult male longtailed macaques (Macaca fascicularis). The first 13 monkeys (A1-B4; Table 1) received grafts as small pieces or slurries. The remaining 11 monkeys (B5-B15; Table 2) received ribbon grafts by preliminary (B5-B8) or well-developed (B9-B12) versions of the ribbon method, or by further experimental versions involving grafts cut to half- length and half-width (B13-B15). Histologica! results and grafting methods, which were reported previously for $\mathrm{B} 1-\mathrm{B} 12 / 8,11 /$, are presented here for A1-A9 and B13-B15. Behavioral data on all 24 monkeys are presented in a separate report $/ 9 /$.

\section{Lesion Surgery}

Each monkey was anesthetized with ketamine/halothane and positioned in a Kopf stereotaxic apparatus with adjustable elevated eyebars and earbars $/ 10 \%$. Ventriculography was performed as described previously /10/ but with iohexol (Sterling Winthrop, Rensselaer NY, 180 $\mathrm{mgI} / \mathrm{ml}$ ) as the radio-opaque contrast medium, injected $19 \mathrm{~mm}$ anterior and $2 \mathrm{~mm}$ left of earbar zero, at the depth (usually $17-21 \mathrm{~mm}$ dorsal) at which the backflow of cerebrospinal fluid moved a small air bubble in the line. Polyvinyl tubing (Bolab, Lake Havasu City AZ, \#BB317-V/3) and a 3-cc syringe were used for injecting iohexol $(0.5$ cc over $30 \mathrm{sec}$ ) through a 23-ga thinwall cannula for a frontal X-ray, and again for a lateral view. Devices for accurately aiming the X-ray and for later adjusting the eyebars have been described previously $/ 10 \%$.

The eyebar adjustment to level the intercommissural line, and the coordinates of the anterior commissure after this adjustment, were calculated from the locations of the commissures in the lateral X-ray /7/. Stereotaxic coordinates in this adjusted space for a set of four tracks to deliver 6-hydroxydopamine $(8 \mu \mathrm{g} / \mu$ l Ringer's solution, with $0.1 \%$ ascorbic acid) to the substantia nigra were $4,6,8$, and $10 \mathrm{~mm}$ behind the anterior commissure and $2 \mathrm{~mm}$ off midline. The deepest level for each track $(5,6,7$, and $7.5 \mathrm{~mm}$ below the anterior commissure, respectively) received $3 \mu \mathrm{l}$ over $3 \mathrm{~min}$, then the needle was raised in $0.5-\mathrm{mm}$ increments, and $1.5 \mu \mathrm{l}$ was injected at each of five more levels. For monkeys A1-A3, 6-hydroxydopamine injections were made along only the three anterior tracks. For monkeys A1-B8, lesions were created via a surgically implanted cranial guide-tube platform /6/; transplants were also made through the platform guide tubes for these monkeys, except B5 and B7 (Table 2). Lesions and transplants in monkeys B9-B15 were created 
directly in surgery under anesthesia, without a platform.

\section{Transplant Surgery: Monkeys A1-A9}

Various tissue-fragment transplant methods were employed in the earliest series (A1-A9, Table 1). Grafts were placed in two caudate sites (A1-A4), two caudate and two putamen sites (A5A8), or two sites in the lateral ventricle (A9). The adrenal gland was cut into pieces and medullary fragments were isolated by piecemeal dissection (A1-A2), or the medulla was separated in one piece from the cortex (A3-A9). Small pieces of medulla were cut with a scalpel (A1), punched out with a sharpened needle (A2), minced with a McIlwain tissue-chopper (Brinkman Instruments, Westbury, NY)(A3-A6), or cross-scalpeled (A7A9). Beveled needles of various sizes were loaded, inserted through platform guide tubes $/ 6 /$, and emptied either by the insertion of a stylet (A1-A2) or by hydraulic pressure (A3-A9). For monkeys A8-A9, micropipette glass with the outer diameter of a 23-ga stainless steel needle (0.64 $\mathrm{mm}$ ) was used for injections, to permit visualization of graft in the needles and to eliminate microscopic metallic flakes observed at many earlier graft sites.

A cell-suspension method was tested in monkey A7. Medullary tissue was cross-scalpeled, gently stirred at $37^{\circ} \mathrm{C}$ for $45 \mathrm{~min}$ in Hanks' balanced salts without calcium or magnesium (HBSS) with $0.02 \%$ deoxyribonuclease, $0.04 \%$ collagenase, and $0.2 \%$ neutral protease, then filtered $(60-\mu \mathrm{m} \mathrm{mesh})$, centrifuged, and re-suspended $/ 2 /$. Cell-suspension $(20-60 \mu 1)$ was placed in each of the four striatal sites, and in one site in the substantia nigra.

Three monkeys received daily nerve growth factor (NGF) injections via platform guide tubes after transplantation. Injections for monkeys A5 and A8 (7S NGF 5 days/wk, $4.17 \mu \mathrm{g}$ in $3 \mu \mathrm{l} /$ day) were placed at two sites, one between the putamen grafts, and the other in the lateral ventricle. Monkey A9 received ventricular injections only (2.5S NGF 5 days/wk, $0.0834 \mu \mathrm{g}$ in $3 \mu \mathrm{l} /$ day).

\section{Transplant Surgery: Monkeys B1-B12}

Techniques for monkeys B1-B12, including post-transplant chronic treatment with NGF, have been described elsewhere 18, 11/: briefly, (i) monkeys B1-B4 received a small amount $(3.5 \mu \mathrm{l})$ of a slurry prepared from minced adrenal tissue; (ii) monkeys B5-B8 received ribbon grafts, but without preservation of the linear form, and (iii) monkeys B9-B12 received full-length, full width ribbon grafts (Tables 1-2).

\section{Transplant Surgery: Monkeys B13-B15}

In monkeys B13-B15, variations of the established method were applied, including finer needles, smaller grafts, a greater concentration of NGF, a larger number of sites, and a longer period between ligation and completion (Table 2). For monkeys B13 and B14, half-ribbons(4-5 mm long) were transplanted to twice as many sites as for B11-B12. Extra-thinwalled needle stock (21XX: 19-ga inner but only 21-ga outer diameter) was fitted with a spinal-needle hub filled with acrylic, and used for half the grafts in each monkey. For the other half, a smaller such needle (25XX: 22-ga inner but only 25 -ga outer diameter) was similarly fitted with a hub. The inner stylet for this needle was 28-ga and the innermost needle was 34-ga. For this smaller set, the 5-mm half-ribbons were split lengthwise. NGF concentrations and chronic treatments were the same for monkey B13 as for monkeys B9-B11, and were 10 times that concentration for monkey B14 (2.5S NGF, $1 \mu \mathrm{g}$ in $60.72 \mu \mathrm{l} /$ day). Monkey B15 received transplants via the smaller $(25 \mathrm{XX})$ needle at thirteen sites throughout the striatum. A \#11 scalpel blade was used for splitting the ribbons lengthwise. The vehicle and chronic ventricular treatment included GM1 ganglioside ( $45.5 \mu \mathrm{g}$ in $60.72 \mu \mathrm{l} /$ day) in HBSS.

\section{Immunohistochemistry}

Grafts were evaluated on the basis of tyrosine hydroxylase $(\mathrm{TH})$ immunohistochemistry by methods previously published $/ 11,12 /$. 
TABLE 1. Techniques: Monkeys A1 - B4

\begin{tabular}{|c|c|c|c|c|c|c|c|c|c|c|c|c|c|}
\hline & A1 & A2 & A3 & A4 & A5 & A6 & A7 & A8 & A9 & B1 & B2 & B3 & B4 \\
\hline $\begin{array}{l}\text { Preparation } \\
\text { Vehicle } \\
\text { Additives }\end{array}$ & $\begin{array}{c}\text { Dissect } \\
\text { Saline } \\
\text {-- }\end{array}$ & $\begin{array}{c}\text { Dissect } \\
\text { Saline } \\
--\end{array}$ & $\begin{array}{c}\text { Cube } \\
\text { Saline } \\
--\end{array}$ & $\begin{array}{c}\text { Cube } \\
\text { Saline } \\
--\end{array}$ & $\begin{array}{l}\text { Cube } \\
\text { Ringers } \\
\text { NGF }\end{array}$ & $\begin{array}{c}\text { Cube } \\
\text { Ringers } \\
\text {-- }\end{array}$ & $\begin{array}{c}\text { Suspend } \\
\text { HBSS } \\
--\end{array}$ & $\begin{array}{l}\text { Mince } \\
\text { Ringers } \\
\text { NGF }\end{array}$ & $\begin{array}{c}\text { Mince } \\
\text { Ringers } \\
\text { NGF }\end{array}$ & $\begin{array}{c}\text { Mince } \\
\text { Ringers } \\
\text { NGF }\end{array}$ & $\begin{array}{c}\text { Mince } \\
\text { Ringers } \\
\text { NGF }\end{array}$ & $\begin{array}{l}\text { Mince } \\
\text { Ringers } \\
\text { NGF }\end{array}$ & $\begin{array}{c}\text { Mince } \\
\text { Ringers } \\
--\end{array}$ \\
\hline $\begin{array}{l}\text { Sites } \\
\text { Platform tubes } \\
\text { Injector }\end{array}$ & $\begin{array}{c}\text { c2 } \\
17 g a \\
19 \text { thin }\end{array}$ & $\begin{array}{c}\text { C2 } \\
\text { 17ga } \\
\text { 22thin }\end{array}$ & $\begin{array}{c}\text { c2 } \\
\text { 17ga } \\
\text { 19thin }\end{array}$ & $\begin{array}{c}\text { c2 } \\
\text { 20thin } \\
\text { 23thin }\end{array}$ & $\begin{array}{l}\text { c2,p2,v1 } \\
16 \mathrm{ga}^{\wedge} \\
23 \mathrm{ga}\end{array}$ & $\begin{array}{l}\text { c2,p2 } \\
16 g a^{\wedge} \\
23 g a\end{array}$ & $\begin{array}{c}\text { c2,p2,s1 } \\
16 g a^{\wedge} \\
23 g a\end{array}$ & $\begin{array}{c}c 2, p 2 \\
16 \mathrm{ga}^{\wedge} \\
23 \text { glass }\end{array}$ & $\begin{array}{c}\text { v2 } \\
16 \mathrm{ga}^{\wedge} \\
23 \text { glass }\end{array}$ & $\begin{array}{c}c 2, p 2 \\
19 \text { thin } \\
19 \text { glass }\end{array}$ & $\begin{array}{c}c 2, p 2 \\
19 \text { thin } \\
19 \text { glass }\end{array}$ & $\begin{array}{c}c 2, p 2 \\
19 \text { thin } \\
19 \text { glass }\end{array}$ & $\begin{array}{c}c 2, p 2 \\
19 \text { thin } \\
19 \text { glass }\end{array}$ \\
\hline $\begin{array}{l}\text { Loading } \\
\text { Ejecting } \\
\text { Volume/Length } \\
\text { Ligation-End }\end{array}$ & $\begin{array}{l}\text { Push } \\
\text { Stylet } \\
\text { na } \\
\text { na }\end{array}$ & $\begin{array}{l}\text { Push } \\
\text { Stylet } \\
\text { na } \\
20\end{array}$ & $\begin{array}{c}\text { Hydr } \\
\text { Hydr } \\
10 \mathrm{ul} \\
30\end{array}$ & $\begin{array}{c}\text { Hydr } \\
\text { Hydr } \\
8 u l \\
45\end{array}$ & $\begin{array}{l}\text { Hydr } \\
\text { Hydr } \\
10 \text { ul }^{*} \\
90\end{array}$ & $\begin{array}{l}\text { Hydr } \\
\text { Hydr } \\
10 \mathrm{ul} \\
71\end{array}$ & $\begin{array}{c}\text { Hydr } \\
\text { Hydr } \\
5-61 \text { ul* } \\
180\end{array}$ & $\begin{array}{c}\text { Hydr } \\
\text { Hydr } \\
20 \mathrm{ul} \\
44\end{array}$ & $\begin{array}{c}\text { Hydr } \\
\text { Hydr } \\
20 \mathrm{ul} \\
48\end{array}$ & $\begin{array}{c}\text { Hydr } \\
\text { Hydr } \\
3.5 \mathrm{ul} \\
72\end{array}$ & $\begin{array}{c}\text { Hydr } \\
\text { Hydr } \\
3.5 \mathrm{ul} \\
80\end{array}$ & $\begin{array}{c}\text { Hydr } \\
\text { Hydr } \\
3.5 \mathrm{ul} \\
83\end{array}$ & $\begin{array}{c}\text { Hydr } \\
\text { Hydr } \\
3.5 \text { ul } \\
72\end{array}$ \\
\hline $\begin{array}{l}\text { Pump cannula } \\
\text { Pump vehicle } \\
\text { Pump additives }\end{array}$ & $\begin{array}{l}-- \\
--\end{array}$ & $\begin{array}{l}-- \\
-- \\
--\end{array}$ & $\begin{array}{l}- \\
- \\
-\end{array}$ & $\begin{array}{l}-- \\
-- \\
--\end{array}$ & $\begin{array}{c}\text { Put, Vent* } \\
\text { Ringers } \\
\text { NGF }\end{array}$ & $\begin{array}{l}-- \\
- \\
-\end{array}$ & $\begin{array}{l}-- \\
-- \\
--\end{array}$ & $\begin{array}{l}\text { Put, Vent* } \\
\text { Ringers } \\
\text { NGF }\end{array}$ & $\begin{array}{l}\text { Vent" } \\
\text { Ringers } \\
\text { NGF }\end{array}$ & $\begin{array}{l}\text { Put^^} \\
\text { Ringers } \\
\text { NGF }\end{array}$ & $\begin{array}{l}\text { Put^^} \\
\text { Ringers } \\
\text { NGF }\end{array}$ & $\begin{array}{c}\text { Put }^{\wedge \wedge} \\
\text { Ringers } \\
--\end{array}$ & $\begin{array}{c}\text { Put^^} \\
\text { Ringers } \\
\text { NGF }\end{array}$ \\
\hline $\begin{array}{l}\text { Lesion-Graft } \\
\text { Graft-Necropsy } \\
\text { Age } \\
\text { Wt }\end{array}$ & $\begin{array}{l}39 \\
29 \\
5.3 \\
2.4\end{array}$ & $\begin{array}{l}59 \\
21 \\
4.5 \\
2.3\end{array}$ & $\begin{array}{l}77 \\
23 \\
5.2 \\
2.7\end{array}$ & $\begin{array}{c}23 \\
15 \\
3 \\
2.9\end{array}$ & $\begin{array}{l}25 \\
21 \\
3.7 \\
2.2\end{array}$ & $\begin{array}{l}35 \\
22 \\
3.6 \\
2.6\end{array}$ & $\begin{array}{c}7 \\
26 \\
3.1 \\
2.3\end{array}$ & $\begin{array}{l}21 \\
23 \\
4.6 \\
2.5\end{array}$ & $\begin{array}{l}23 \\
59 \\
3.4 \\
2.2\end{array}$ & $\begin{array}{l}35 \\
16 \\
2.5 \\
2.4\end{array}$ & $\begin{array}{l}34 \\
34 \\
4.5 \\
2.8\end{array}$ & $\begin{array}{l}51 \\
34 \\
4.3 \\
2.6\end{array}$ & $\begin{array}{l}51 \\
49 \\
4.3 \\
2.4\end{array}$ \\
\hline $\begin{array}{l}\text { Est. colls } \\
\text { Positive sites }\end{array}$ & $\begin{array}{l}973 \\
2 / 2\end{array}$ & $\begin{array}{l}126 \\
1 / 2\end{array}$ & $\begin{array}{c}0 \\
0 / 2\end{array}$ & $\begin{array}{c}0 \\
0 / 2\end{array}$ & $\begin{array}{l}489 \\
2 / 4\end{array}$ & $\begin{array}{l}58 \\
1 / 4\end{array}$ & $\begin{array}{c}0 \\
0 / 5\end{array}$ & $\begin{array}{l}10 \\
1 / 4\end{array}$ & $\begin{array}{c}0 \\
0 / 2\end{array}$ & $\begin{array}{l}62 \\
3 / 4\end{array}$ & $\begin{array}{l}13 \\
0 / 4\end{array}$ & $\begin{array}{c}9 \\
1 / 4\end{array}$ & $\begin{array}{c}0 \\
0 / 4\end{array}$ \\
\hline
\end{tabular}

TABLE 2. Techniques: Monkeys B5 - B15

\begin{tabular}{|c|c|c|c|c|c|c|c|c|c|c|c|}
\hline & B5 & B6 & B7 & B8 & B9 & B10 & B11 & B12 & B13 & B14 & B15 \\
\hline $\begin{array}{l}\text { Preparation } \\
\text { Vehicle }\end{array}$ & $\begin{array}{l}\text { Razors } \\
\text { Ringers }\end{array}$ & $\begin{array}{l}\text { Razors } \\
\text { Ringers }\end{array}$ & $\begin{array}{l}\text { Razors } \\
\text { HBSS }\end{array}$ & $\begin{array}{l}\text { Razors } \\
\text { HBSS }\end{array}$ & $\begin{array}{l}\text { Razors } \\
\text { HB/Gara }\end{array}$ & $\begin{array}{l}\text { Razors } \\
\text { HB/Gara }\end{array}$ & $\begin{array}{l}\text { Razors } \\
\text { HB/Gara }\end{array}$ & $\begin{array}{l}\text { Razors } \\
\text { HB/Gara }\end{array}$ & $\begin{array}{l}\text { Razors } \\
\text { HB/Gara }\end{array}$ & $\begin{array}{l}\text { Razors } \\
\text { HB/Gara }\end{array}$ & $\begin{array}{l}\text { Razors } \\
\text { HB/Gara }\end{array}$ \\
\hline Additives & - & - & NGF & NGF,Lam & NGF & NGF & NGF & NGF & NGF & NGF & Gang \\
\hline Sites & $c 2, p 2$ & $c 2, p 2$ & $c 2, p 2$ & $c 2, p 2$ & $c 2, p 2$ & $c 2, p 2$ & $c 2, p 2, s 1$ & $c 2, p 2, s 1$ & $c 5, p 5, s 2$ & $c 5, p 5, s 2$ & $\infty, p 7$ \\
\hline $\begin{array}{l}\text { Platform tubes } \\
\text { Outer Injector } \\
\text { Inner Injector }\end{array}$ & $\begin{array}{c}- \\
19 g a \\
22 g a\end{array}$ & $\begin{array}{l}16 g a \\
19 g a \\
22 g a\end{array}$ & $\begin{array}{c}-. \\
19 g a \\
22 g a\end{array}$ & $\begin{array}{l}16 g a \\
19 g a \\
22 g a\end{array}$ & $\begin{array}{c}\text { 19ga } \\
\text { 22/28ga }\end{array}$ & $\begin{array}{c}-- \\
19 g a \\
22 / 28 g a\end{array}$ & $\begin{array}{c}19 g a \\
22 / 28 g a\end{array}$ & $\begin{array}{c}\overline{-} \\
\text { 19ga } \\
22 / 28 g a\end{array}$ & $\begin{array}{c}-- \\
21 X X^{*} \\
22 / 28 g a\end{array}$ & $\begin{array}{c}-- \\
21 X X^{\star} \\
22 / 28 g a\end{array}$ & $\begin{array}{c}-- \\
25 X X \\
28 / 34 g a\end{array}$ \\
\hline $\begin{array}{l}\text { Loading } \\
\text { Ejecting } \\
\text { Volume } \\
\text { Ligate-End }\end{array}$ & $\begin{array}{c}\text { Push } \\
\text { Stylet } \\
\text { na } \\
57\end{array}$ & $\begin{array}{l}\text { Push } \\
\text { Stylet } \\
\text { na } \\
47\end{array}$ & $\begin{array}{c}\text { Push } \\
\text { Stylet } \\
5.0 \mathrm{~mm} \\
50\end{array}$ & $\begin{array}{c}\text { Push } \\
\text { Stylet } \\
7.7 \mathrm{~mm} \\
37\end{array}$ & $\begin{array}{c}\text { Pull } \\
\text { Reveal } \\
9.8 \mathrm{~mm} \\
64\end{array}$ & $\begin{array}{c}\text { Pull } \\
\text { Reveal } \\
8.1 \mathrm{~mm} \\
53\end{array}$ & $\begin{array}{l}\text { Pull } \\
\text { Reveal } \\
8.2 \mathrm{~mm} \\
65\end{array}$ & $\begin{array}{c}\text { Pull } \\
\text { Reveal } \\
11.1 \mathrm{~mm} \\
70\end{array}$ & $\begin{array}{c}\text { Pull } \\
\text { Reveal } \\
5.0 \mathrm{~mm} \\
144\end{array}$ & $\begin{array}{c}\text { Pull } \\
\text { Reveal } \\
4.5 \mathrm{~mm} \\
164\end{array}$ & $\begin{array}{c}\text { Pull } \\
\text { Reveal } \\
3.9 \mathrm{~mm} \\
157\end{array}$ \\
\hline Pump cannula & -- & -- & Vent $^{\wedge}$ & -- & Vent^ & Vent^ & Vent & Vent & Vent & Vent & Vent \\
\hline Pump vehicle & -- & -- & HBSS & -- & HB/Gara & HB/Gara & HB/Gara & HBSS & HB/Gara & HB/Gara & HB/Gara \\
\hline Pump additives & -- & -- & NGF & -- & NGF & NGF & NGF & -- & NGF & NGF & Gang \\
\hline Lesion-Graft & 27 & 15 & 51 & 52 & 38 & 45 & 15 & 14 & 72 & 77 & 27 \\
\hline Graft-Necropsy & 35 & 28 & 28 & 28 & 27 & 28 & 56 & 52 & 57 & 56 & 33 \\
\hline Age & 4.9 & 2 & 3.8 & 3.5 & 4.7 & 4.5 & na & na & 3.2 & 3.8 & na \\
\hline$W t$ & 2.7 & 2.5 & 2.3 & 2.3 & 2.8 & 3.5 & 2.2 & 2.1 & 2 & 2.3 & 2.2 \\
\hline Est. œ $œ$ & 1740 & 399 & 5047 & 1910 & 3035 & 25865 & 15281 & 49008 & 2564 & 5760 & 6977 \\
\hline Positive sites & $3 / 4$ & $4 / 4$ & $4 / 4$ & $3 / 4$ & $4 / 4$ & $4 / 4$ & $5 / 5$ & $5 / 5$ & $9 / 10$ & $10 / 10$ & $9 / 13$ \\
\hline
\end{tabular}


TABLES 1 and 2

Preparation. Dissect: pieces cut away with scalpel (monkey A1) or punched out with tubing (monkey A2). Cube: tissue chopper ( $200 \mu \mathrm{m}$ for 19 thin injector, $100 \mu \mathrm{m}$ for 23 thin injector). Suspend: cross-scalpeling and enzyme incubation. Mince: cross scalpeling, with scalpels (monkeys A8-A9) or carbide blades (B1-B4). Razors: razor-blade assemblage. A1-A9: prepared in glass petri dish. B1-B15: prepared on roughened Teflon plate. B5-B15: ribbons stenciled on wax sheet.

Vehicle, Additives. HBSS: Hanks' balanced salts without calcium or magnesium. HB/Gara: HBSS with antibiotic Garamycin (0.2 $\mathrm{mg} / \mathrm{ml})$. NGF: Nerve growth factor (see text for concentrations). Lam: Laminin ( $20 \mu \mathrm{g} / \mathrm{ml}$. Gang: GM1 gangloside $(0.75 \mu \mathrm{g} / \mu \mathrm{l})$.

Sites. c2: two sites in head of caudate. p2: two sites in putamen. v1: one site in ventricle. s1: one site in and above substantia nigra.

Platform tubes. Gauge of guide-tube implanted in platform. $\wedge:$ 19-ga tubing was lowered acutely through 16-ga to top of striatum to guide injector to site. *: guide-tubes stopped at top of skull; skull drilled just before transplant.

Injector. Gauge of injector containing graft. *half the sites were grafted with 25XX Outer, 28/34-ga Inner Injector.

Loading, Ejecting Push: primarily using Dumont forceps to push tissue in at the tip. Hydr: simple suction to draw slurry into injector or eject into brain. Pull: 22/28-ga or 28/34-ga used to pull ribbon into injector in standard method. Stylet: pushed with stylet to eject into brain. Reveal: pulled up outer needle first, exposing graft to brain in linear conformation.

Volume. *: A5: $10 \mu 1$ in each striatal site, $18 \mu 1$ in ventricle; A7: 61, 42, 34, 23, and $5 \mu 1$ in four striatal sites and one nigral site respectively. na: not available.

Ligation-end. Time from ligation of adrenal vessel to end of final transplantation (min).

Pump Cannula. Put: needle implanted near putamen graft sites. Vent: needle implanted in lateral ventricle on treated side. *: no pump, sites injected on weekdays. $\wedge \wedge$ : Model 2002 (2-wk pump) implanted in platform. $\wedge$ : Model 2002 (2-wk pump) implanted subcutaneously on back. All others: Model 2ML4 (4-wk) implanted subcutaneously on back.

Lesion-Graft, Graft-Necropsy. Time from lesion to graft and from graft to necropsy (days).

Age. Age in years at necropsy.

Wt. Weight in kilograms at necropsy.

Est. Cells. Direct cell-counts for grafts with few survivors; estimates for large graft.

Positive sites. Number of sites with immunoreactive tissue/total number of target sites.

\section{Measurement}

Because it was assumed that transplants needed to be set in place as quickly as possible, photographs or careful measurements of each ribbon were not taken. The length, width, and thickness of each ribbon graft were roughly estimated before transplant for monkeys B9-B12, but only the length for monkeys B13-B15 (Table 3). Cellcounts for surviving graft tissue in histological sections were also problematic. As noted for fluorescence-labeled grafts in rodents $/ 16 /$, and confirmed in the present material, densely clustered medullary graft cells are difficult to count accurately and their large numbers in ribbon grafts made direct cell-counts impractical. Quantitative assessment was nonetheless necessary for com- paring the results of graft techniques. For these reasons, cell numbers were estimated on the basis of approximate cell counts in a few small grafts and rough areal estimates of all grafts, as follows. For most monkeys, one $40-\mu \mathrm{m}$ coronal section was immunostained every 80 to $160 \mu \mathrm{m}$. The plane of section and the linear ribbon grafts were both perpendicular to the horizontal plane of the intercommissural line. Often most of the linear extent of a graft was represented in one or two sections, although portions appeared in sections as far as 250-300 $\mu \mathrm{m}$ anterior or posterior. Skipping discontinuities, and focusing on the sections containing the most positive tissue, I estimated the total dorsoventral length and the average maximal width along this length for each graft. Then the graft was modeled in concept as a narrow cylinder 
TABLE 3. Graft Dimensions

\begin{tabular}{|c|c|c|c|c|c|c|c|c|c|c|c|}
\hline \multirow[t]{2}{*}{ Monkey } & \multicolumn{2}{|c|}{ Viable Length:Striatum } & \multicolumn{2}{|c|}{ Viable Cells:Striatum } & \multirow[t]{2}{*}{ Site } & \multirow{2}{*}{$\begin{array}{l}\text { Average } \\
\text { Width }\end{array}$} & \multirow{2}{*}{$\begin{array}{l}\text { Tot Viable } \\
\text { Length }\end{array}$} & \multirow{2}{*}{$\begin{array}{r}\text { Tot Viable } \\
\text { Cells }\end{array}$} & \multirow{2}{*}{$\begin{array}{l}\text { Percent } \\
\text { In STR }\end{array}$} & \multicolumn{2}{|c|}{ Original Ribbon } \\
\hline & CAU & PUT & CAU & PUT & & & & & & Length 1 & Thickness \\
\hline \multirow[t]{4}{*}{ B9 } & 2.33 & 4.60 & 468 & 2568 & Caudate & 0.06 & 2.20 & 461 & $100 \%$ & 12.3 & $0.8 \times 0.8$ \\
\hline & & & & & Caudate & 0.03 & 0.13 & 7 & $100 \%$ & 7.9 & $0.8 \times 0.8$ \\
\hline & & & & & Putamen & 0.03 & 2.10 & 110 & $100 \%$ & 9.5 & $0.6 \times 0.8$ \\
\hline & & & & & Putamen & 0.13 & 2.50 & 2458 & $100 \%$ & 6.7 & $0.8 \times 0.8$ \\
\hline \multirow[t]{4}{*}{ B10 } & 4.89 & 0.00 & 8236 & 0 & Caudate & 0.16 & 6.40 & 9533 & $39 \%$ & 7.9 & $1.0 \times 0.6$ \\
\hline & & & & & Caudate & 0.08 & 7.00 & 2291 & $0 \%$ & 7.9 & $1.0 \times 0.4$ \\
\hline & & & & & Caudate & 0.18 & 4.70 & 8860 & $51 \%$ & 8.3 & $1.0 \times 0.6$ \\
\hline & & & & & Putamen & 0.13 & 5.70 & 5182 & $0 \%$ & 8.3 & $1.0 \times 0.4$ \\
\hline \multirow[t]{5}{*}{ B11 } & 6.47 & 1.46 & 9635 & 494 & Caudate & 0.16 & 3.70 & 5511 & $97 \%$ & 9.1 & $0.8 \times 0.6$ \\
\hline & & & & & Caudate & 0.16 & 3.00 & 4468 & $96 \%$ & 7.5 & $1.0 \times 0.4$ \\
\hline & & & & & Putamen & 0.05 & 1.50 & 218 & $40 \%$ & 8.7 & $1.0 \times 0.8$ \\
\hline & & & & & Putamen & 0.09 & 3.75 & 1767 & $23 \%$ & 8.7 & $1.0 \times 0.8$ \\
\hline & & & & & Nigra & 0.10 & 5.70 & 3316 & $0 \%$ & 7.1 & $1.0 \times 0.8$ \\
\hline \multirow[t]{5}{*}{ B12 } & 7.11 & 0.42 & 10634 & 755 & Caudate & 0.18 & 7.70 & 13720 & $62 \%$ & 11.1 & $1.0 \times 0.8$ \\
\hline & & & & & Caudate & 0.13 & 5.20 & 4727 & $45 \%$ & 11.1 & $1.0 \times 0.8$ \\
\hline & & & & & Putamen & 0.18 & 5.30 & 9444 & $8 \%$ & 11.1 & $1.0 \times 0.8$ \\
\hline & & & & & Putamen & 0.13 & 7.00 & 6364 & $0 \%$ & 11.1 & $1.0 \times 0.8$ \\
\hline & & & & & Nigra & 0.21 & 5.75 & 14753 & $0 \%$ & 11.1 & $1.0 \times 0.8$ \\
\hline \multirow[t]{10}{*}{ B13 } & 4.35 & 4.00 & 186 & 298 & Caudate* & 0.03 & 2.00 & 105 & $100 \%$ & 5 & na \\
\hline & & & & & Putamen & 0.06 & 0.65 & 136 & $100 \%$ & 5 & na \\
\hline & & & & & Caudate* & 0.03 & 2.50 & 91 & $86 \%$ & 5 & na \\
\hline & & & & & Putamen & 0.04 & 1.75 & 125 & $100 \%$ & 5 & na \\
\hline & & & & & Caudate* & 0.02 & 0.20 & 3 & $100 \%$ & 5 & na \\
\hline & & & & & Putamen & 0.00 & 0.00 & 0 & $100 \%$ & 5 & na \\
\hline & & & & & Caudate" & 0.07 & 0.70 & 200 & $0 \%$ & 5 & na \\
\hline & & & & & Putamen & 0.02 & 1.60 & 37 & $100 \%$ & 5 & na \\
\hline & & & & & Nigra* & 0.05 & 2.60 & 378 & $0 \%$ & 5 & na \\
\hline & & & & & Nigra & 0.08 & 4.00 & 1489 & $0 \%$ & 5 & na \\
\hline \multirow[t]{10}{*}{ B14 } & 7.12 & 2.00 & 2900 & 216 & Caudate & 0.04 & 1.60 & 149 & $67 \%$ & 4.5 & na \\
\hline & & & & & Caudate" & 0.06 & 3.00 & 628 & $100 \%$ & 4.5 & na \\
\hline & & & & & Caudate & 0.14 & 1.70 & 1939 & $100 \%$ & 4.5 & na \\
\hline & & & & & Caudate* & 0.02 & 0.50 & 7 & $50 \%$ & 4.5 & na \\
\hline & & & & & Caudate & 0.06 & 1.10 & 230 & $100 \%$ & 4.5 & na \\
\hline & & & & & Putamen* & 0.03 & 0.80 & 42 & $100 \%$ & 4.5 & na \\
\hline & & & & & Caudate & 0.08 & 1.10 & 410 & $0 \%$ & 4.5 & na \\
\hline & & & & & Putamen" & 0.05 & 1.20 & 175 & $100 \%$ & 4.5 & na \\
\hline & & & & & Nigra & 0.15 & 1.00 & 1309 & $0 \%$ & 4.5 & na \\
\hline & & & & & Nigra* & 0.09 & 1.85 & 872 & $0 \%$ & 4.5 & na \\
\hline \multirow[t]{12}{*}{ B15 } & 3.21 & 8.58 & 1989 & 4543 & Caudate* & 0.10 & 1.50 & 873 & $100 \%$ & 5 & na \\
\hline & & & & & Putamen" & 0.00 & 0.00 & 0 & $100 \%$ & 3 & na \\
\hline & & & & & Caudate* & 0.07 & 0.70 & 200 & $30 \%$ & 5 & na \\
\hline & & & & & Putamen* & 0.10 & 1.80 & 1047 & $100 \%$ & 4.5 & na \\
\hline & & & & & Caudate* & 0.00 & 0.00 & 0 & $100 \%$ & 4.5 & na \\
\hline & & & & & Putamen" & 0.04 & 0.80 & 74 & $100 \%$ & 2.5 & na \\
\hline & & & & & Caudate* & 0.11 & 1.50 & 1056 & $100 \%$ & 2.5 & na \\
\hline & & & & & Putamen" & 0.09 & 0.50 & 236 & $100 \%$ & 2.5 & na \\
\hline & & & & & Caudate* & 0.00 & 0.00 & 0 & $100 \%$ & 4 & na \\
\hline & & & & & rutamen & 0.10 & 1.00 & 582 & $100 \%$ & 4 & na \\
\hline & & & & & Putamen" & 0.10 & 210 & 1222 & $75 \%$ & 4 & na \\
\hline & & & & & Putamen" & 0.10 & 2.90 & 1687 & $100 \%$ & 5 & na \\
\hline
\end{tabular}


TABLE 3

Viable length. Cumulative DV length of portions of ribbon containing viable tissue ( $\mathrm{mm}$ ).

Viable cells. Estimated number of TH-immunoreactive cells. (For monkey B9 only one or two sections were available for each graft and substantial portions may not be documented.)

Site. *: half-width ribbon grafted at this site.

Average width. Mean ML width of viable portions of graft.

Percent in STR. Percentage of graft tissue appearing in the striatum.

Original ribbon. Dimensions before grafting. B13-B15: only length was measured, to shorten surgery time.

of the corresponding height and diameter. Countable cells in a $40-\mu \mathrm{m}$ circular cross-section through the cylinder were estimated at 400 per $0.1 \mathrm{sq} \mathrm{mm}$, on the basis of approximate cell-counts of small grafts. As the mean diameter of a cell must be added to the thickness of the section to compensate for double-counting surface cells $/ 1 /$, the cross-section was considered to be $55 \mu \mathrm{m}$ thick. The number of cells per cross-section, multiplied by the number of $55-\mu \mathrm{m}$ cross-sections in the cylinder, gave the estimate of the number of cells in the graft (Tables 1-3).

\section{RESULTS}

\section{Monkeys A1-A4}

The best graft tissue surviving after 3-4 weeks was a compact $\mathrm{TH}$-immunoreactive fragment of medulla in the lateral ventricle of monkey A1 (Fig. 1a), but another graft, containing fewer cells, was found in the caudate parenchyma of A2 (Fig. 1b). Tracks also contained microscopic black flecks resembling metallic filings, apparently introduced by the injectors (Fig. 1b). After 3 weeks, no viable immunoreactive graft tissue could be detected in monkey A3, and only a few questionable cells in monkey A4 after 2 weeks; the objects were lightly stained and had ragged morphology (Fig. 2a).

\section{Monkeys A5-A9}

There was a cluster of viable immunoreactive cells near the NGF injection site in the putamen of monkey A5 (Fig. 2b); monkey A6 was not treated with NGF, but a small cluster survived in the putamen (Fig. 2c). Although these findings confirmed that parenchymal grafts could survive, the numbers of cells were very small. Metallic filings were again observed in graft sites, even near the successful grafts (Fig. 2b). Immnohistochemical examination revealed no active graft tissue from the cell-suspension grafts in A7, and only a few cells, of poor morphology, along each track in monkey A8; the best example was a cluster of cells in a putamen track near the NGF injections (Fig. 2d). No viable tissue from the ventricular grafts (monkey A9) could be detected.

\section{Monkeys B1-B12}

Results for monkeys B1-B12, summarized in Tables 1-3, have been described and illustrated elsewhere $/ 8,11 /$. A section through a portion of a nigral graft, not previously displayed, illustrates the striking viability of the grafts in B9-B12 (Fig. $3 b)$. Table 3 provides a quantitative comparison of ribbon-grafted sites in monkeys B9-B15.

\section{Monkeys B13-B15}

For these subjects, ribbon grafts were prepared as described previously $/ 8,11 /$, except that all grafts were cut to approximately $5 \mathrm{~mm}$ length, half that of earlier ribbon grafts, and some were additionally bisected to half-width. Most graft sites contained less viable tissue than expected, even considering the smaller size of the original ribbon. Of the caudate grafts in B13 (half-width), three of four contained small clumps of immunoreactive cells. Three of four putamen grafts (full-width), contained larger clusters, but fell short of half the 
length of grafts in monkeys B9-B12. One nigral graft (half-width) had no labeled tissue; the other (full-width) was a good graft (Fig. 3a), but not on the scale of earlier full-length, full-width grafts (Fig. 3b). In monkey B14 the caudate grafts (fullwidth) were generally thick but short, ranging over 1-2 $\mathrm{mm}$ in length. The putamen grafts (halfwidth) were thinner. Both nigral grafts, made 2.5 $\mathrm{h}$ after the adrenal was tied off, were viable and 2 $\mathrm{mm}$ long (Fig. 4a), among the best grafts in this monkey. Monkey B15 received 13 grafts, all half- width. Six of seven grafts in the putamen contained substantial numbers of cells, in clusters 100-200 $\mu \mathrm{m}$ wide. Most of these clusters were short (1-2 mm), but the last two grafts, for which the time spent in vehicle before grafting was longest, were 3-4 mm (Fig. 4b), close to the length of the original ribbons. In the caudate, two of six grafts were similar to those in the putamen, but the other four contained scattered cells at best, most of them in the corpus callosum above the caudate and ventricle; some graft tissue may have been deposited in the ventricle, leaving little trace.

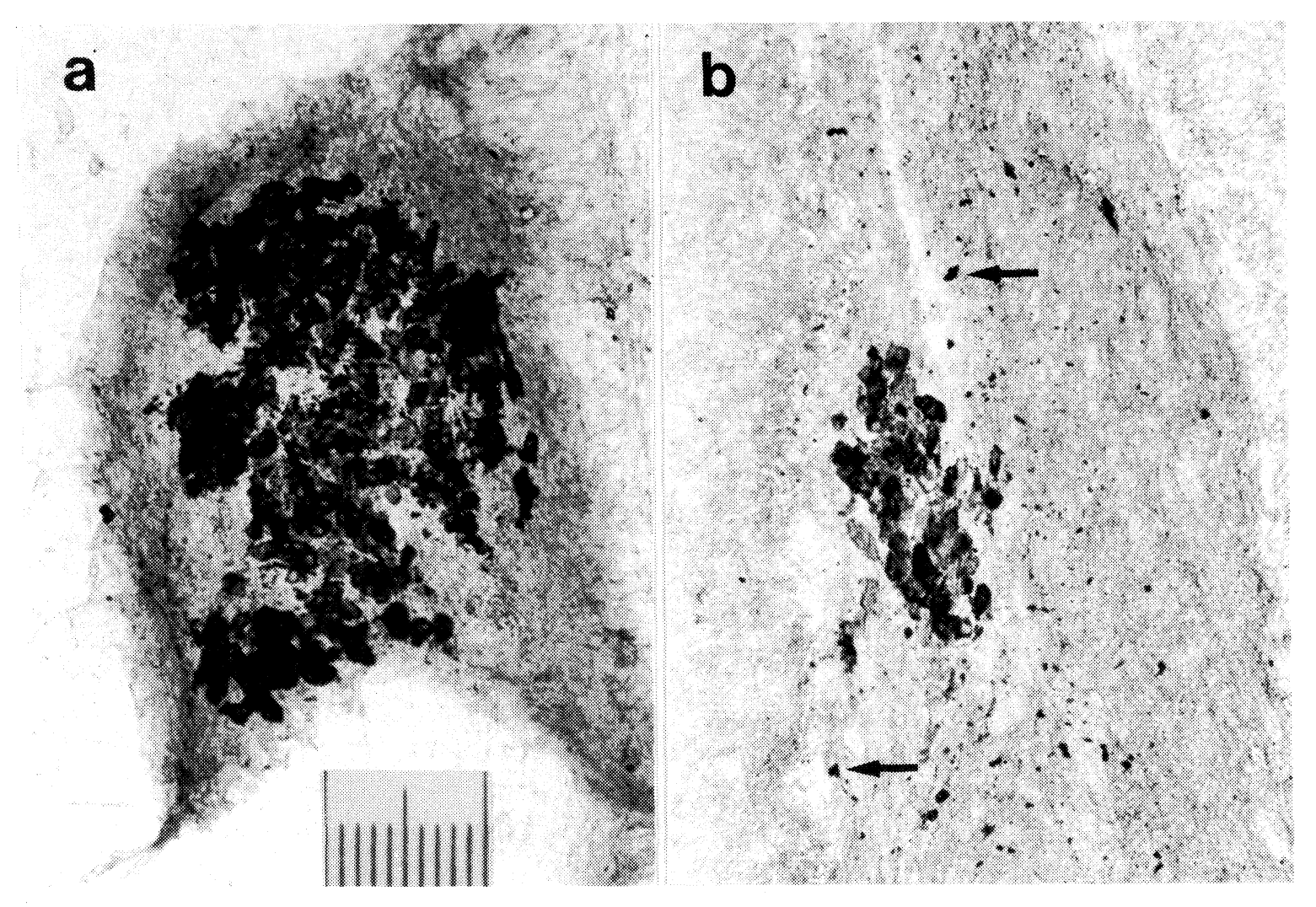

Fig. 1: Small, dense cluster of TH-immunoreactive cells in the ventral tip of the lateral ventricle in monkey A1 (a), in the caudate nucleus in monkey A2 (b). Small arrows indicate metallic flecks found at the graft site (b). Magnification 200x, for all figures except Fig. 5a. Scale represents $100 \mu \mathrm{m}$ in $10-\mu \mathrm{m}$ increments. All figures display the largest example of a graft site in a given monkey, oriented with the dorsal end up. 


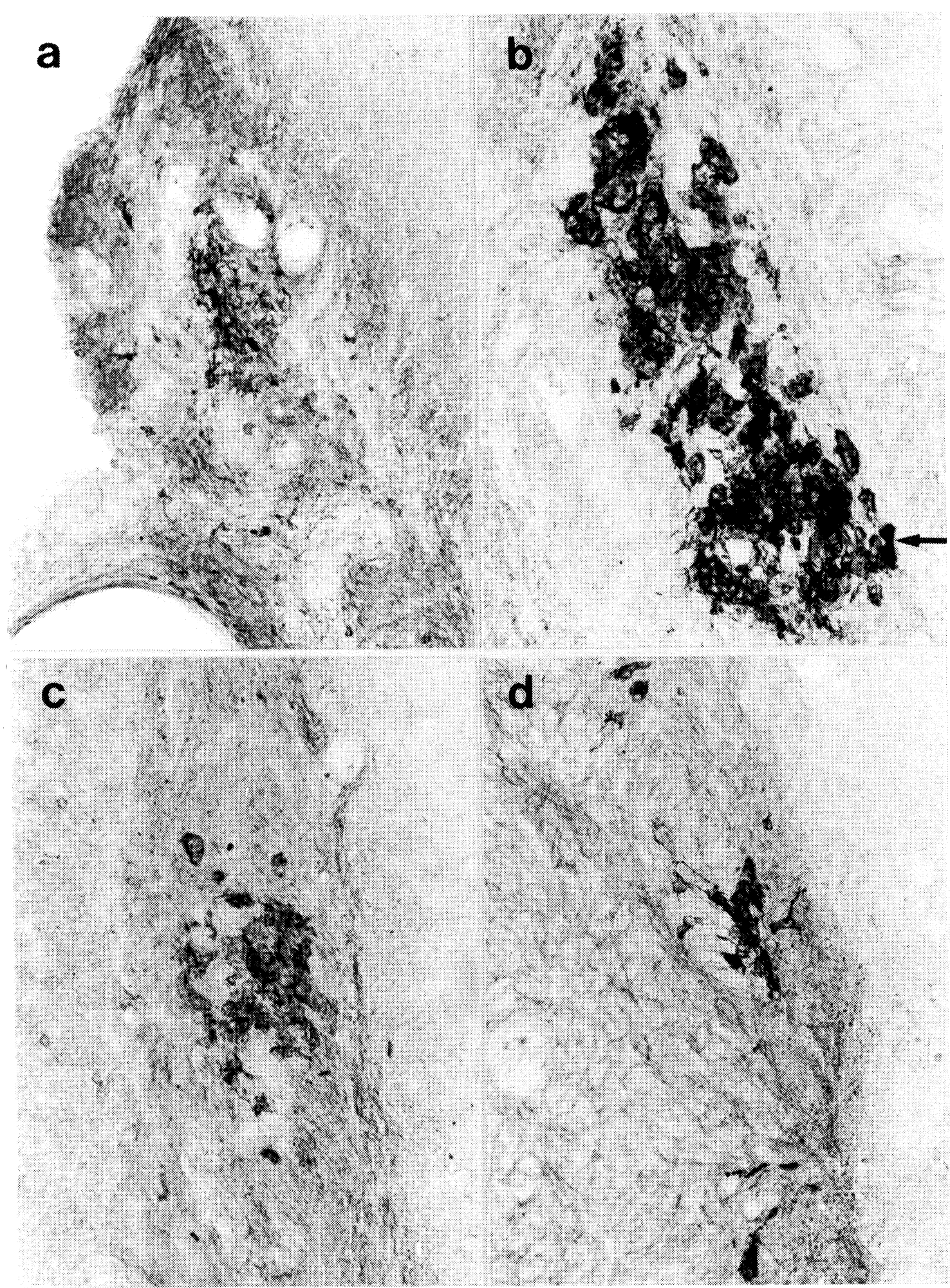

Fig. 2: a. Questionable objects in monkey A4, specifically stained (never observed in non-immune serum control sections), but lacking typical cellular morphology. $b$. Best graft in monkey A5, located in the putamen near the NGF injection site. Small arrow marks a metallic fleck at the site. c and d. Best clusters of cells in monkeys A6 (putamen) and A8 (putamen). 




Fig. 3: Nigral grafts in monkeys B13 (a) and B12 (b).

\section{Post-surgical Tissue}

Full-width, half-length graft tissue from an additional monkey that died a few hours after transplantation, owing to complications involving anesthesia, supplements data on graft viability. Little graft tissue adhered to brain tissue after frozen-sectioning, but a few of the small strips were successfully processed and mounted. They document ribbons of thickness up to $600 \mu \mathrm{m}$ (Fig. 5a). Most adrenal cells were stained relatively lightly, but cells along the edges were darker and very compressed, and cells near the top and bottom were also very darkly stained (Fig. 5b).

\section{DISCUSSION}

Several biological and technical aspects of medullary grafting have been discussed previously $18,11 /$. Other issues are raised by the additional experiments presented in this report, including the preparation and size of grafts, incubation prior to grafting, proper use of injection needles, and 


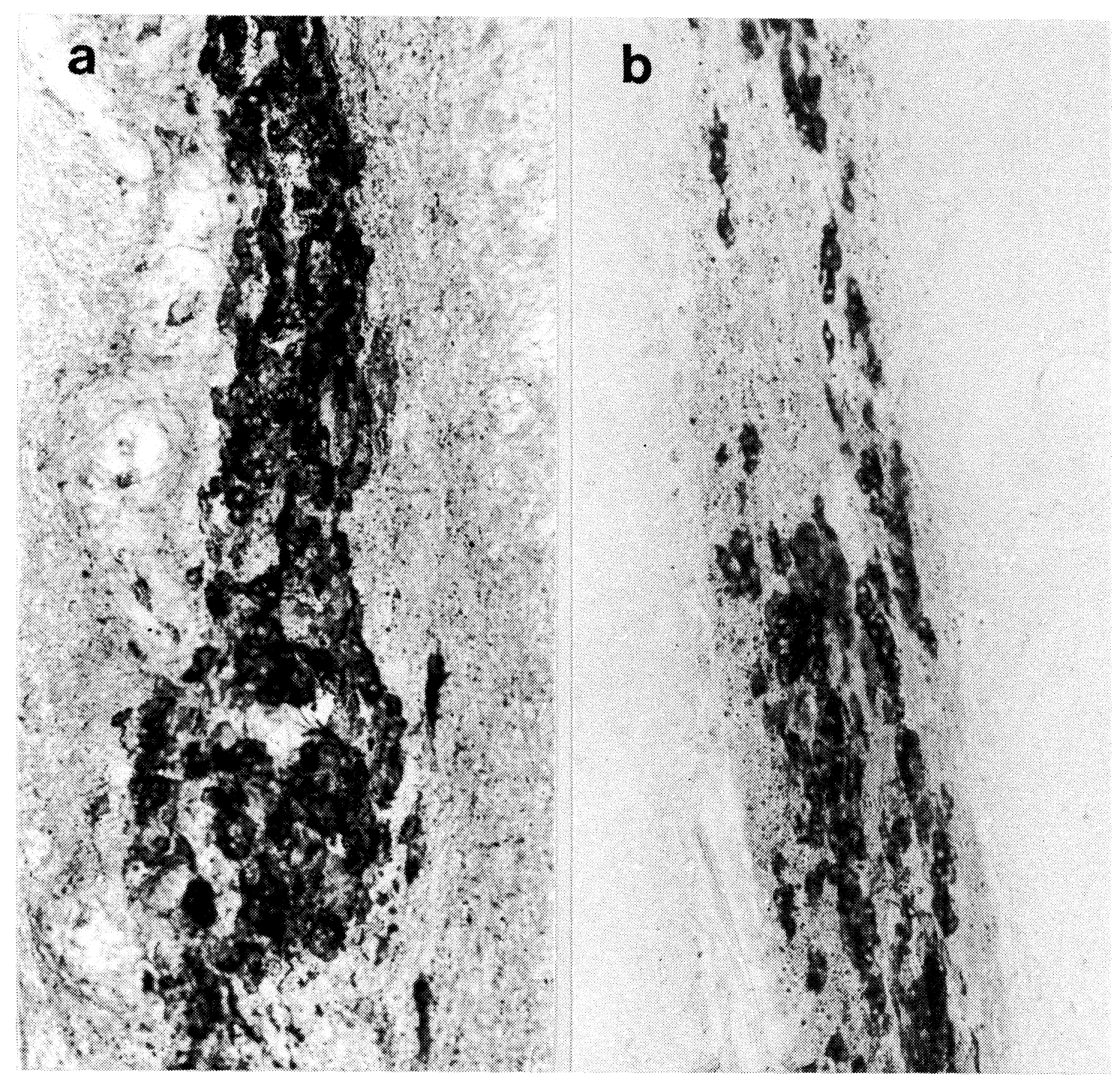

Fig. 4: Best grafts in monkeys B14 (a, nigra) and B15 (b, putamen), each made more than $2.5 \mathrm{~h}$ after adrenal ligation.

variation of the adrenal medulla among individuals.

\section{Preparation}

Early tissue-fragment methods (Table 1: monkeys A1-B4) met with minimal, capricious success by comparison with the ribbon method. These results were roughly similar to those of several other published accounts of adrenal graft histology in monkeys /4, 20, 28/. Ribbon grafts, on the other hand, consistently yielded large amounts of viable, immunoreactive tissue (Table 3: monkeys B9-B15). Even so, proportional viability of full-size ribbons was well under 50\%; halflength, half-width ribbons, moreover, yielded grafts of smaller proportional viability than fullwidth ribbons, in the monkeys that received both sizes (B13-B14). The cut surface therefore appears to be less viable than the internal portion of a ribbon; cutting cells at this surface and compressing cells just below may seriously com- 


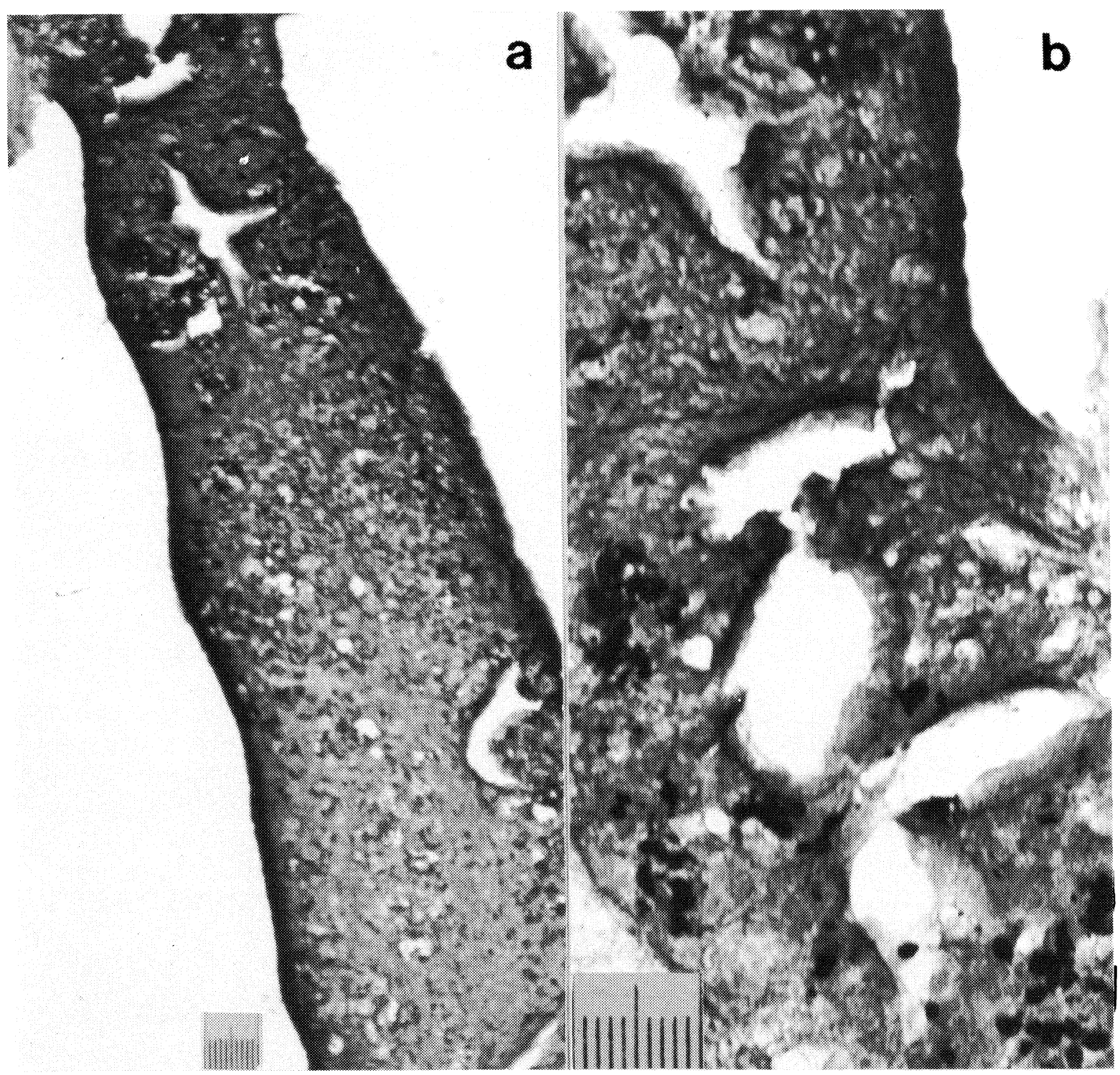

Fig. 5: a, Low-magnification view (85x) of adrenal ribbon a few hours after transplantation. Staining intensity is lower and morphology less distinct than that of intact adrenal medullary tissue or viable graft tissue 4-8 wks after transplantation. b, High-magnification view (200x) of the upper end of the same ribbon, displaying signs of physical disruption and intensely stained small round objects without nuclei.

promise viability near the cut surface. Other vulnerable regions may be the dorsal end of the ribbon, stressed by the suction of the 22/28-ga needle that grasps it as the ribbon is loaded, and the cross-cut end of a half-length ribbon; even the full-width ribbons in monkeys B13 and B14 gave a smaller proportional yield, presumably because they were cut to half-length. The acute post-sur- gical graft (Fig. 5) is consistent with these interpretations; the compressed appearance of cells at the periphery of the ribbon directly after transplantation may have been due in part to stresses of cutting and loading. Damage at the periphery may also compromise the interior of a ribbon to some extent, since anastomosis with brain vessels must initially take place at the 
stressed surface. In addition, ribbons of medulla can be stretched slightly in vitro, and internal portions of the graft may be stretched or broken during loading.

Physical damage of these kinds may be sufficient to reduce the original ribbon to the narrower, variably discontinuous column of viable tissue seen in histological sections. Areas in ribbon grafts that did survive and were immunoreactive were similar in appearance to intact adrenal medulla /11/, so that a loss of certain tissue components throughout the graft, such as connective tissue, blood vessels, or a subset of chromaffin cells, is unlikely to account for the diminution. Nor could the apparent loss of well over half the transplanted tissue be attributed to small natural discontinuities of TH-immunoreactivity owing to embedded islands of zona reticulata (ZR) tissue, connective tissue, large vessels, and sinusoids.

Various earlier methods of dicing tissue (A1B4) involved more serious problems, which were evident during the surgery as well as during later histological evaluation, such as the adherence of tissue to blades, dehydration of tissue fragments, inconsistent sizes of adrenal pieces, and inconsistent amounts of vehicle added during dissection. None of these efforts led to large viable grafts, and in retrospect, dicing tissue to a small size for stereotaxic needles probably compromises the capacity for reperfusion, leaving less integrable material for viable grafts. Excess cutting, whether in the preparation of ribbon grafts or tissue-fragment grafts, may diminish viability. Only the ribbon method described above led to large-scale viability. It may be noted that the one prior report of behavioral effects possibly attributable to adrenal grafts in monkeys involved large pieces $(1 \times 1 \times 5 \mathrm{~mm})$ of medulla $/ 32 /$.

\section{Vehicle}

The interval between ligation of adrenal vessels and introduction of ribbons to transplant vehicle is brief (5-10 $\mathrm{min})$. Once the ribbons are in vehicle, results clearly indicate that surgical velocity is not a significant factor. Ribbons that remained in HBSS with NGF or ganglioside at room temperature for $2-2.5 \mathrm{~h}$ made strongly viable grafts (B13-B15). This has several important implications: the number of grafts to be made is probably not time-limited; adrenalectomy can be completed before the monkey is placed in the stereotaxic device; several hours may be available for incubation with agents to improve graft function, and there is time for detailed measurement and photography to document each ribbon before grafting.

NGF was included in the vehicle and incubation medium for all ribbon-graft surgeries except one; GM1 ganglioside was substituted in that case (B15), and the viable grafts in this subject suggest that NGF incubation is not critical. The salt solution was HBSS for all successful grafts, but no test has compared it directly with saline, lactated Ringer's, or other media. Chronic ventricular NGF treatment is not essential for viability, as judged by results in monkey $\mathrm{B} 12$, which received chronic HBSS alone, and monkey B15, which received chronic GM1 ganglioside; both animals had somewhat larger viable grafts than others receiving comparable ribbons. The concentration of NGF chronically infused into the ventricle for monkey B14 was 10 times that used for B9-B11 and B13, but did not induce neuronal morphology in the grafted cells.

\section{Injection needles}

Neither a single stainless steel needle (A1-A7) nor glass tubing (A8-B4) made a satisfactory injector, nor did any of these experiments yield results favoring one needle size over another. Early ribbon grafts (B5-B8) were mostly loaded by being pushed in from the tip and injected by being pushed out with a 22-ga inner needle. Such grafts were found at the bottom of the track and contained a small proportion of viable tissue, in contrast to later ribbon grafts. It is clearly preferable to load and to eject as described for the ribbon method /8/. Spinal-needle injector assemblies of various sizes (19-ga, 21XX, or $25 \mathrm{XX}$; monkeys B9-B15) yielded substantial survival of medullary tissue. By comparison with the 19-ga spinal needle, the 21XX needle provided a smaller outer diameter for the same inner diameter. The thinner injector $(25 \mathrm{XX})$ did not appear to increase viability or to encourage the formation of proces- 
ses by chromaffin cells. The increased amount of graft trimming and handling involved in loading the $25 \mathrm{XX}$ needle probably compromised viability. This needle also bent more easily, which made handling more difficult and decreased stereotaxic accuracy. For all needles, softening the internal edge of the bevel eased loading. An acid wash before each surgery, and reuse of the same needle many times, practically eliminated the occurrence of metallic filings at graft sites for ribbon grafts.

\section{Variation}

Biological variation may have a substantial influence on grafts. For example, the largest grafts in the present material (B12) were also the largest pre-transplant ribbons from the largest adrenal medulla. Variability also affects the ease of preparation and the degree of contamination with ZR tissue. The adrenal gland varied among the 24 subjects in size, shape, color, ZR and medullary thickness, strength of adrenal vein musculature, and tenacity of tissue holding medulla and cortex together at their interface. For one of the first subjects, for example, even though the edges of the intact gland were not trimmed or the cortex intentionally retracted, as became standard practice for later monkeys, the piecemeal removal of external adipose, vascular, and connective tissue from the intact gland pulled away most of the cortex from the medulla without further effort. For other subjects the adherence between these layers was stronger, and although the orderly dissection scheme as described $/ 8 /$ has been much more reliable than piecemeal dissection, the tissue itself is nonetheless highly variable among individuals in this regard.

Corresponding variation was also observed in a microscopic analysis of a large sample of glands from rhesus macaques $/ 22 /$. In that study, there were variations in the amount of connective tissue and amorphous intercellular material in the ZR, in the amount of acellular connective tissue forming an incomplete "capsule" between the ZR and the medulla, and in the numbers of pigmented cells in both ZR and underlying connective tissue. These elements were not observed in fetal or very young monkeys, but otherwise they were unrelated to age. Islands of ZR cells are known to be present in human adrenal medulla $/ 21 /$, and islands of $\mathrm{TH}$-immunonegative cells have been observed in monkey adrenal medulla $/ 11 /$; the frequency and distribution of these islands, like those of norepinephrine and epinephrine islands $/ 13 /$, are likely to vary among individuals.

\section{Comparison with Other Reports}

The ribbon method, like the stereotaxic cograft method recently described $/ 25 /$, has been relatively reliable in terms of graft viability. Adrenal medullary grafts made by the ribbon method displayed remarkable viability after eight weeks survival. The viable immunoreactive graft tissue, however, did not account for the majority of the volume of the original ribbon. Experiments with smaller, more sculpted ribbons have indicated that much of the loss of chromaffin tissue occurs at the cut surfaces and ends of the ribbons. This may also be an important observation in the context of clinical medullary grafts that have already been made, even though very different methods have been used. Further experimentation with medullary ribbon grafts in non-human primates should focus on reducing the stress of preparation at the cut margins of the ribbon.

Owing to biological variability, no two graft subjects can be strictly comparable. Individual variation in the raw material of adrenal autografts has undoubtedly contributed to graft variability in the present ribbon-graft subjects (B9-B15). The observation that medullary ribbons can be left in vehicle for at least 2.5 hours, and possibly much longer, gives time for detailed photography and measurement of each graft, so that the influence of such factors on viability can now be explored.

A principal advantage of stereotaxic grafting is flexibility in the choice and number of graft sites. If the reversal of the behavioral effects of nigral lesions in monkeys requires the replacement of catecholamines in the striatum, it is important to determine how many grafts and which locations are necessary for reversing various effects. A behavioral assay, presented in the accompanying report along with data from all the subjects described above, initiates this process for ribbon grafts $/ 9 \%$. 
Aside from the subjects described in the present report, a total of 34 other non-human primate subjects have received adrenal grafts $/ 4,20,25,28$, $30,32,33 /$. Histological data have been reported for 30 of these subjects. A variety of methods have been employed, with the sample sizes for any one method averaging 2.5 animals (range 1 to 4 ). Subjects have been intact ( 5 subjects), lesioned by IV-N-methylphenyltetrahydropyridine (MPTP) (2), or unilaterally by intracarotid MPTP (18) or intracerebral 6-OHDA (5). Grafts have been made stereotaxically (7), stereotaxically via a silver carrier (4), stereotaxically after transcallosal exposure of the caudate (4), or into a cavity in the caudate nucleus made transcallosally after an interhemispheric approach (9) or after a transcortical approach (6). A wide range of viable tissue has resulted from these experiments, from none (9) or fewer than 100 chromaffin cells/site (7) to an average of 5500-6000/site (6) /25/; for 8 subjects only qualitative descriptions were given (a "thin rim" and "small rests" $/ 30 /$; TH immunoreactivity "in cells within the confines of the graft" and DBH immunoreactivity in "less than $3 \%$ of the estimated total number of grafted catecholaminergic cells" $/ 32 /$ ).

For a variety of reasons, the present report as well as these previous reports concern case studies of individual subjects or very small numbers of subjects for any given set of techniques. These studies are breaking a trail, not building a highway. A full-scale critical test of a single given set of techniques and graft sites would not yet be appropriate. From a perspective based on non-human primate research, the time has not yet arrived for clinical applications. It is important to examine closely the techniques, histology, and behavior reported for each animal in order to use the most promising techniques for further studies.

\section{ACKNOWLEDGEMENTS}

I wish to thank Greg Anderson, Joel Cummings, Doug Hasund, and Tom Susor for technical assistance, and other members of the Primate Center staff for animal care and veterinary services. Iohexol for ventriculography was kindly provided by the Sterling-Winthrop Research In- stitute (Rensselaer, NY). Support for this work came in part from NIH grant RR00166 to the University of Washington Regional Primate Research Center, and primarily from NIH (NINDS) grant RO1 NS25724 for Neuron Transplant Therapy.

\section{REFERENCES}

1. Abercrombie M. Estimation of nuclear population from microtome sections. Anat Rec 1946; 94: 239-247.

2. Ali-Osman F, Beltz PA. Optimization and characterization of the capillary human tumor clonogenic cell assay. Cancer Res 1988;48: 715-724.

3. Apuzzo MLJ, Neal JH, Waters CH, Appley AJ, Boyd SD, Couldwell WT, Wheelock VH, Weiner LP. Utilization of unilateral and bilateral stereotaxically placed adrenomedullary-striatal autografts in Parkinsonian humans: Rationale, techniques, and observations. Neurosurgery 1990; 26: 746-757.

4. Bankiewicz KS, Plunkett RJ, Kopin IJ, Jacobowitz DM, London WT, Oldfield EH. Transient behavioral recovery in hemiparkinsonian primates after adrenal medullary allografts. Prog Brain Res 1988; 78: 543549.

5. Bischoff S, Scatton B, Korf J. Dopamine metabolism, spiperone binding and adenylate cyclase activity in the adult rat hippocampus after ingrowth of dopaminergic neurons from embryonic implants. Brain Res 1979; 179: 77-84.

6. Dooley DJ, Dubach M, Blake PH, Bowden DM. A chronic, stereotaxic guide-tube platform for intracranial injections in macaques. $J$ Neurosci Meth 1981; 3: 385-396.

7. Dubach M. Accurate stereotaxic injection by radially curved injection needles. Neurosurgery 1991; 29 : 144 149.

8. Dubach M. Adrenal medulla "ribbon" grafts in nonhuman primates: Transplant method. J Neurosci Meth 1991; 39: 19-28.

9. Dubach M. Behavioral effects of adrenal medullary transplants in non-human primates. J Neur Transplant Plast 1992; 3: 97-114.

10. Dubach MF, Tongen VC, Bowden DM. Techniques for improving stereotaxic accuracy in Macaca fascicularis. J Neurosci Meth 1985; 13: 163-169.

11. Dubach M, German DC. Extensive survival of chromaffin cells in adrenal medulla "ribbon" grafts in the monkey neostriatum. Exp Neurol 1990; 110: 167-180.

12. Dubach M, Schmidt R, Bowden DM, Kunkel D, Martin R, German DC. Primate neostriatal neurons containing tyrosine hydroxylase: immunohistochemical evidence. Neurosci Lett 1987; 75: 205-210.

13. Eranko O. Fluorescing islets, adrenaline and noradrenaline in the adrenal medulla of some common 
laboratory animals. Ann Med Exp Biol Fenn 1955; 33 : 278-290.

14. Frank F, Sturiale C, Gaist G, Manetto V. Adrenal medullary autograft in human brain for Parkinson's disease. Acta Neurochir (Wien) 1988; 94: 162-163.

15. Freed CR, Breeze RE, Rosenberg NL, Schneck SA, Wells TH, Barrett JN, Grafton ST, Huang SC, Eidelberg D, Rottenberg DA. Transplantation of human fetal dopamine cells for Parkinson's disease:

16. Freed WJ. Functional brain tissue transplantation: reversal of lesion-induced rotation by intraventricular substantia nigra and adrenal medulla grafts, with a note on intracranial retinal grafts. Biol Psychiat 1983; 18: 1205-1267.

17. Freed WJ. Adrenal medulla grafts in animals. Science 1988; 241: 275.

18. Freed WJ, Cannon-Spoor E, Krauthamer E. Intrastriatal adrenal medulla grafts in rats. J Neurosurg 1986; 65: 664-670.

19. Freed WJ, Perlow MJ, Karoum FK, Seiger A, Olson L, Hoffer B, Wyatt RJ. Restoration of dopaminergic function by grafting of fetal rat substantia nigra to the caudate nucleus: long-term behavioral, biochemical, and histochemical studies. Ann Neurol 1980; 8: 510519.

20. Hansen JT, Kordower JH, Fiandaca MS, Jiao S-S, Notter MFD, Gash DM. Adrenal medullary autografts into the basal ganglia of Cebus monkeys: Graft viability and fine structure. Exp Neurol 1988a; 102: 65-75.

21. Hansen JT, Notter MFD, Okawara S-H, Gash DM. Organization, fine structure, and viability of the human adrenal medulla: considerations for neural transplantation. Ann Neurol 1988b; 24: 599-609.

22. Holmes RL. The adrenal glands of Macaca mulatta, with special reference to the cortico-medullary zone. J Anat 1968; 103: 471-477.

23. Hurtig H, Joyce J, Sladek JR Jr, Trojanowski JQ. Postmortem analysis of adrenal-medulla-to-caudate autograft in a patient with Parkinson's disease. Ann Neurol 1989; 25: 607-614.

24. Jankovic J, Grossman R, Goodman C, Pirozzolo F, Schneider L, Zhu Z, Scardino P, Garber AJ, Jhingran
SG, Martin S. Clinical, biochemical, and neuropathologic findings following transplantation of adrenal medulla to the caudate nucleus for treatment of Parkinson's disease. Neurology 1989; 39: 1227. 1234.

25. Kordower JH, Fiandaca MS, Notter MFD, Hansen JT, Gash DM. NGF-like trophic support from peripheral nerve for grafted rhesus adrenal chromaffin cells. J Neurosurg 1990; 73: 418-428.

26. Lindvall $\mathrm{O}$, Bjorklund $\mathrm{A}$. Transplantation strategies in the treatment of Parkinson's disease: experimental basis and clinical trials. Acta Neurol Scand 1989; 126: 197-210.

27. Madrazo I, Drucker-Colin R, Diaz V, Martinez-Mata J, Torres C, Becerril JJ. Open microsurgical autograft of adrenal medulla to the right caudate nucleus in two patients with intractable Parkinson's disease. New Engl J Med 1987; 316: 831-834.

28. Morihisa JM, Nakamura RK, Freed WJ, Mishkin M, Wyatt RJ. Adrenal medulla grafts survive and exhibit catecholamine-specific fluorescence in the primate brain. Exp Neurol 1984; 84: 643-653.

29. Peterson DI, Price ML, Small CS. Autopsy findings in a patient who had an adrenal-to-brain transplant for Parkinson's disease. Neurology 1989; 39: 235-238.

30. Plunkett RJ, Bankiewicz KS, Cummins AC, Miletich RS, Schwartz JP, Oldfield EH. Long-term evaluation of hemiparkinsonian monkeys after adrenal autografting or cavitation alone. J Neurosurg 1990; 73: 918-926.

31. Schmidt RH, Ingvar $M$, Lindvall $O$, Stenevi $U$, Bjorklund A. Functional activity of substantia nigra grafts reinnervating the striatum: Neurotransmitter metabolism and $\left[{ }^{14}-\mathrm{C}\right] 2$-deoxy-D-glucose autoradiography. J Neurochem 1982; 38: 737-748.

32. Watts RL, Bakay RAE, Herring CJ, Sweeney KM, Colbassani HJ, Mandir A, Byrd LD, Iuvone PM. Preliminary report on adrenal medullary grafting and cografting with sural nerve in the treatment of hemiparkinson monkeys. Prog Brain Res 1990; 82: 581-591.

33. Wyatt RJ, Morihisa JM, Nakamura RK, Freed WJ. Transplanting tissue into the brain for function: Use in a model of Parkinson's disease. Res Publ Assn Res Nerv Ment Dis 1986; 64: 199-208. 

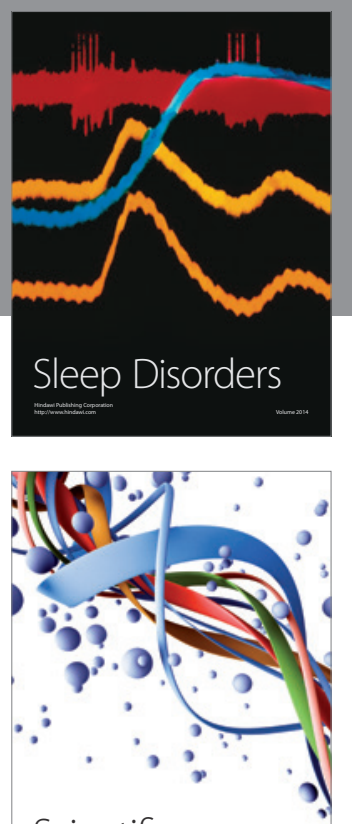

Scientifica
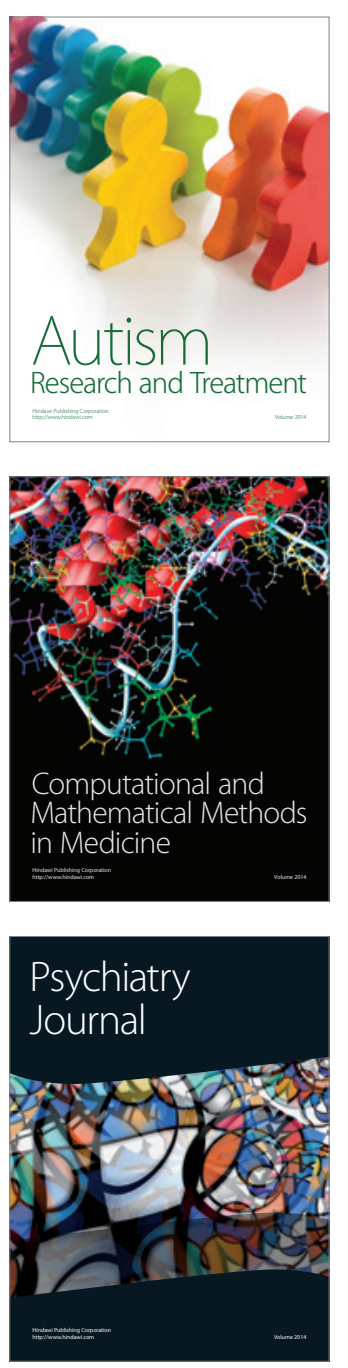
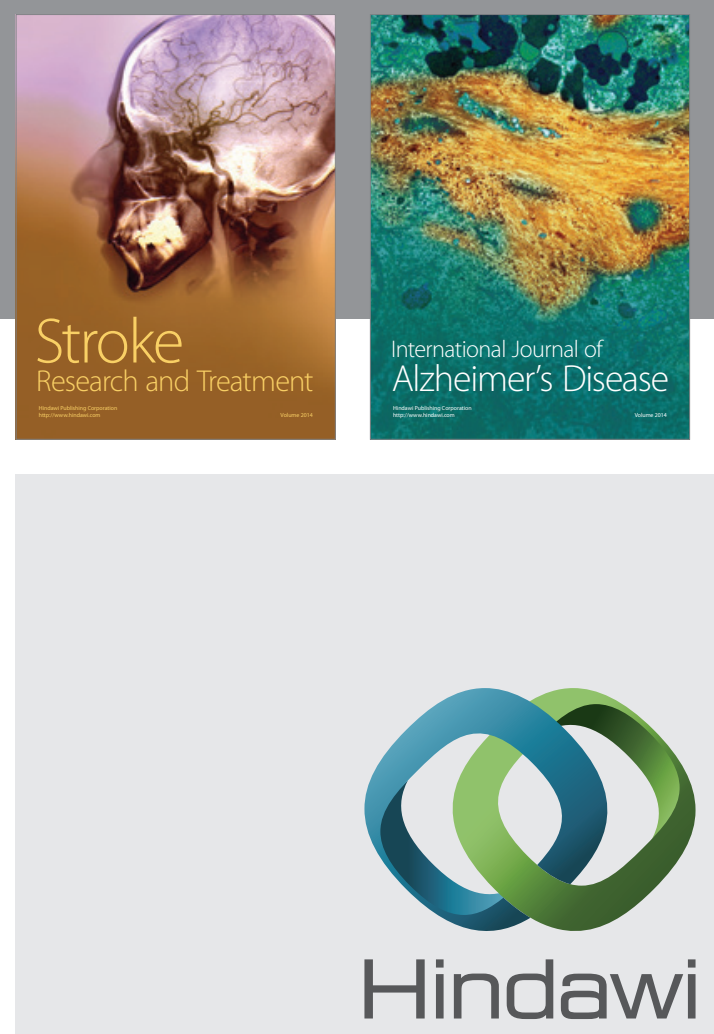

Submit your manuscripts at

http://www.hindawi.com
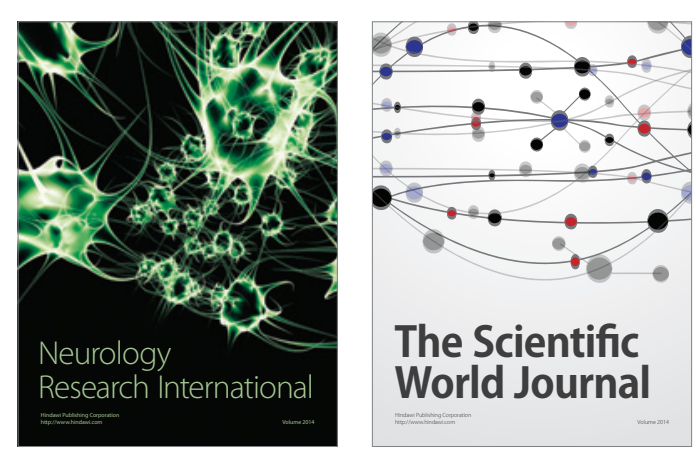

The Scientific World Journal

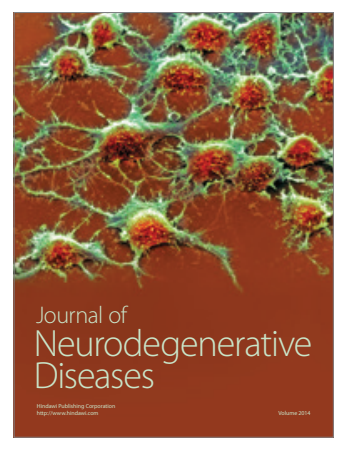

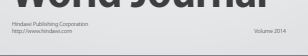

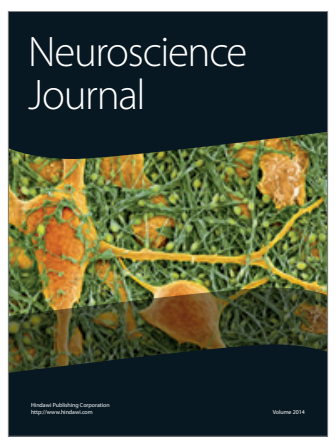

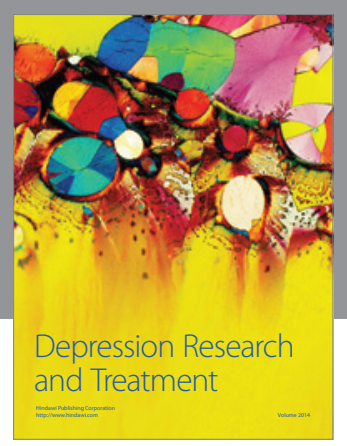
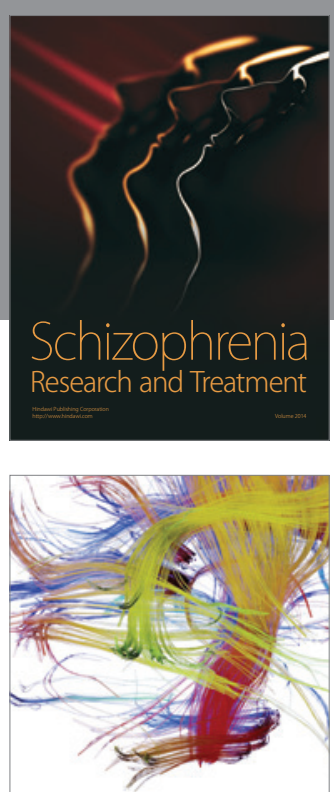

Brain Science

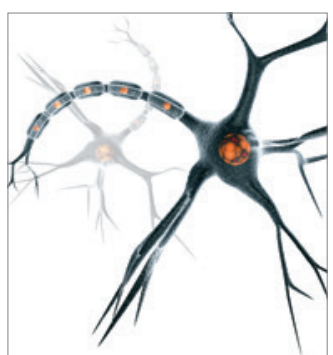

Neural Plasticity
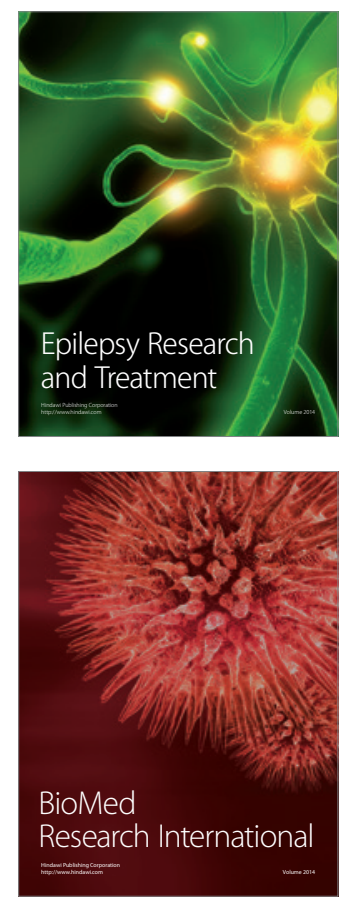

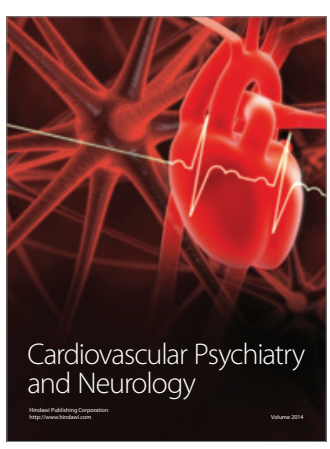

Parkinson's

Disease
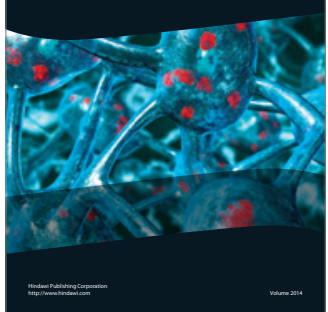